Usage, Usability, and Utility of 3D City Models, 03003 (2012)

DOI: $10.1051 / 3 \mathrm{u} 3 \mathrm{~d} / 201203003$

(c) Owned by the authors, published by EDP Sciences, 2012

\title{
Microscopic transport model animation visualisation on KML base
}

\author{
I. Yatskiv and M. Savrasovs
}

Transport and Telecommunication Institute, LV-1019, Lomonosova street 1, Riga, Latvia

\begin{abstract}
By reading classical literature devoted to the simulation theory it could be found that one of the greatest possibilities of simulation is the ability to present processes inside the system by animation. This gives to the simulation model additional value during presentation of simulation results for the public and authorities who are not familiar enough with simulation. That is why most of universal and specialised simulation tools have the ability to construct 2D and 3D representation of the model. Usually the development of such representation could take much time and there must be put a lot forces into creating an adequate 3D representation of the model. For long years such well-known microscopic traffic flow simulation software tools as VISSIM, AIMSUN and PARAMICS have had a possibility to produce 2D and 3D animation. But creation of realistic 3D model of the place where traffic flows are simulated, even in these professional software tools it is a hard and time consuming action. The goal of this paper is to describe the concepts of use the existing on-line geographical information systems for visualisation of animation produced by simulation software. For demonstration purposes the following technologies and tools have been used: PTV VISION VISSIM, KML and Google Earth.
\end{abstract}

\section{INTRODUCTION}

One of the big advantages of the simulation is a possibility to visualise processes, which happen in the system, and to present output data in graphical form. Usually talking about animation and visualisation of the results in graphical form we must think about dimension. At the moment mostly $2 \mathrm{D}$ and $3 \mathrm{D}$ are used for animation and visualisation purposes. It is connected with human brain limitations to perceive graphical data. In [1] the questions connected with transformation operations, production criteria and user criteria when we are talking about the use of 3D are discussed. All popular professional universal simulation tools like ExtendSim, Arena, etc. could produce both 2D and 3D animation during simulation. Going deeper, possibility of animation and visualisation in 2D and 3D now is included in the list of requirements for the simulation software [2]. Visualisation in transport modelling is an essential aspect for:

- multidimensional character of input and output data;

- in such view data usually presented for public and transport authorities for decision-making.

The one direction of R\&D in this area is connected with visualisation the process or results. The American Association of State Highway and Transportations Officials in July, 2003 published a guide for Transportation Agency "Visualization in Transportation" [3]. There are discussed and determined 2D, 3D and 4D visualisation in transportation.

One of the most famous researches in the spatial-temporal data visualisations has been developed by R. Chapleau supervision. For example, Chapleau, Morency, et al. used 3D GIS techniques to visualise the Montreal travel survey [4, 5]. Schönfelder and Axhausen [6] developed a number of techniques for visualising travel survey data, including the construction of a confidence ellipse. Y. Srinivasan and M-J. Kraak [7] described the visualisations of spatial-temporal patterns in public transport data.

This is an Open Access article distributed under the terms of the Creative Commons Attribution License 2.0, which permits unrestricted use, distribution, and reproduction in any medium, provided the original work is properly cited. 


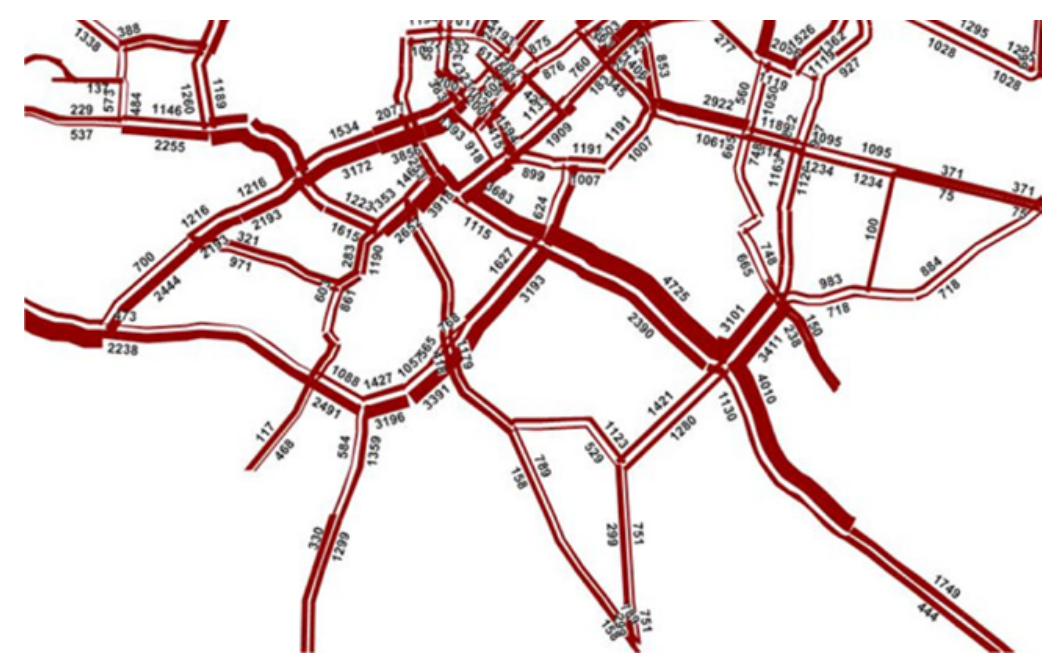

Figure 1. The example of data presentation in macroscopic models (traffic volumes).

Hixon, et al. [8] developed a framework for integrating, analysing and visualising traffic data using GIS in the UK. Kimpel [9] discussed the visualisation in transit planning on the example of bus transit and using the performance information. According to him, transit agencies are faced with a set of internal problems including data collection; post-processing; integration with backend systems; limited budgets; inadequate training of in-house staff; inadequate fusion with existing geographic information systems (GIS); incompatible file formats and other operational issues. Walker [10] noted one additional important thing: the greatest impediment to the use of visualisation as a communication tool is the credibility factor.

The mentioned-above point, push us to propose some tools for traffic simulation software. Of course, software has its own architecture and object hierarchy that is why the example will be presented on the base of PTV VISION VISSIM software. This software is well known across traffic simulation professionals and is one of the leaders in this field. Whereas the offered approach of data visualisation can help to solve some limitation of the existing modern simulation software. These limitations are as follows: on browsing modelling results without simulation software; on data sharing across group of users; on data presentation. The approach on KML basis, developed by the authors, offers a solution for presenting microscopic simulation results and dynamic control over data presentation and observation.

\section{VISUALISATION AND TRANSPORT MODELLING}

In transport modelling we can distinguish microscopic, mesoscopic and macroscopic models. Mesoscopic and macroscopic transport models mostly use 2D visualisation because of high aggregation level of the output data [11]. Most of the well-known macroscopic simulation tools like EMME/2, VISUM, CUBE, etc. present output data in 2D. The example of such presentation could be seen on Figure 1.

How we could extend visualisation possibilities of macroscopic tools by using on-line geographical information systems (GIS) like Google Earth has been described in the paper [12]. In the present paper we are going to show the possibility of application the same concepts for microscopic simulation tools. The work on development of microscopic transport models usually requires a construction of 3D representation. It is connected with a wish to have representative materials for the public and authorities. Another reason is an application of the 3D animation for model validation. 


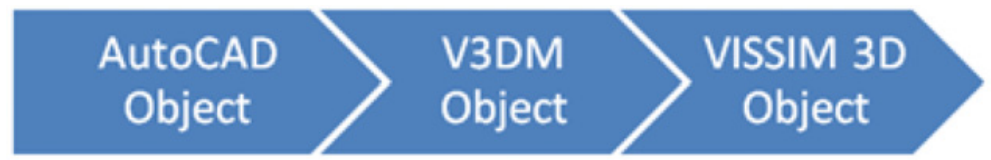

Figure 2. VISSIM 3D object creation procedure.

In case studies examples of application 3D animation in project are shown. Simulation model by itself was developed in professional simulation software called PTV VISION VISSIM. The PTV VISION VISSIM software has its own tools for 3D model representation construction. The primary $3 \mathrm{D}$ representation is done automatically. It means that VISSIM have a library of 3D vehicles, which are used in 3D animation. Also, the movement paths are represented according to the user defined attributes, like 2D location of the road plus one attribute that shows a location of object on z-coordinate. The obtained 3D animation is very simple and do not have a presentation force. That is why additional 3D objects must manually put in to model. VISSIM differ two types of 3D object, the so-called static 3D object and dynamic 3D objects. Static 3D object means that object cannot change its state during time, dynamic means that some of the object parameters could be changed. The examples of static object used in VISSIM are buildings, public transport stops, trees, etc. As dynamic objects vehicles, traffic lights, barriers, and so on could be treated. VISSIM have its own libraries of predefined static and dynamic 3D objects. But if none standard objects are required for visualisation the additional module of VISSIM called V3DM should be used to create user objects. Unfortunately, the proposed tool is very simple and cannot be used to create object with complex geometry but it allows importing already created object from AutoCAD. The direct import from AutoCAD to the VISSIM is forbidden, therefore as a closed graphical format is used. So the following procedure was used for new 3D objects creation (see Figure 2).

For example, in the project, which Transport and Telecommunication Institute (TTI) fulfilled in 2007 [13], approximately 20 new 3D objects were created for urban transport node with 3 level flyover simulation. Most of them are building with applied textures (presented with simple geometrical form).

The largest and biggest 3D object created in this project is a flyover by itself. By using architectural schemas AutoCAD the object has been created. The object is complex and includes the following sub objects: road, pylons, fences, street lights and curbs. Finally, textures are applied for each sub objects and object is transformed into V3DM object. V3DM object cannot be divided into sub objects and presented as one object. Finally the V3DMobject is posted into VISSIM and combined with already created road surface in VISSIM. The Figure 3 demonstrates the created 3D object of the flyover (inside the model).

The motivation behind this point is connected with the fact that currently used visualisation possibilities in simulation tools are concentrated on constructing 3D environment inside the tools. These lead developers to use standard predefined 3D objects, which do not create realistic representation of the environment. Another possibility, which is widely implemented in microscopic simulation tools, includes export of trace file into graphic processing software like Autodesk 3ds Max. But still developer is faced a problem of 3D realistic environment creation. So, to conclude the 3D environment creation in modern microscopic traffic simulation tools is a hard and time consuming procedure, which could take even more time than for model development by itself and experimentation.

To solve this disadvantage we are proposing idea of using on-line GIS, like Google Earth, which at the moment have a lot of cities presented in 3D. Google Earth has already predefined 3D environment that could be used to demonstrate graphical representation of the model. So, microscopic models are developed on the high level of detail, that is why, it is almost a standard to have a possibility to animate and visualise in 2D and 3D [14]. 


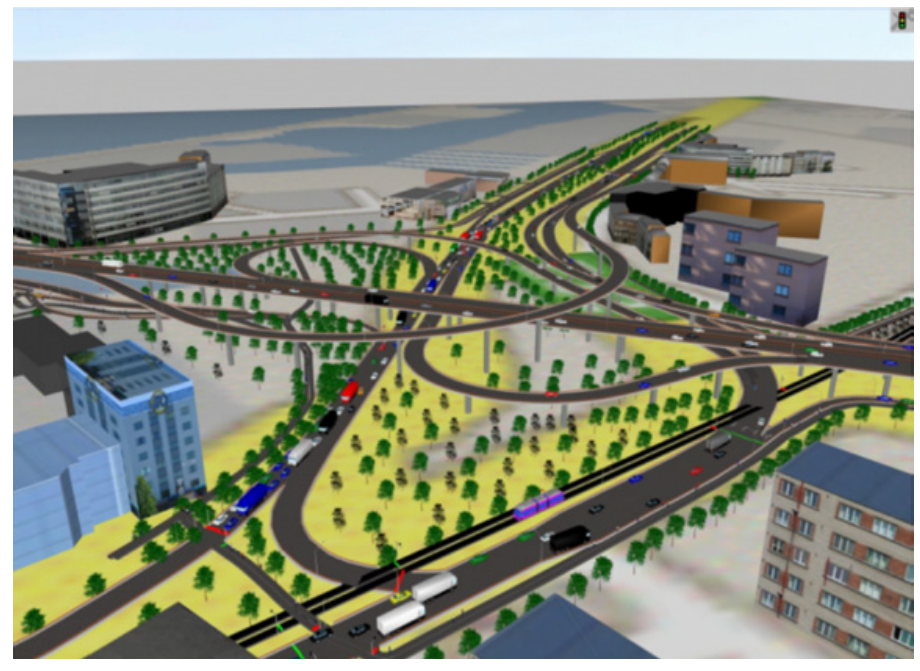

Figure 3. Created 3D objects in transport model.

\section{KEYHOLE MARKUP LANGUAGE}

As it has been mentioned before the main idea of the proposed concepts is to use GIS for visualisation of animation. That is why we should have a tool how we could interact with the GIS; at the moment most popular tool is KML. Not looking on popularity, analysis of the described above task limitations of the traffic flow simulation software on data presentation, and visualisation the decision to use Keyhole Markup Language was taken.

Keyhole Markup Language (KML) is an XML-based language schema for expressing geographic annotation and visualisation on Internet-based, two-dimensional maps and three-dimensional Earth browsers. KML has been developed for use with Google Earth, which is originally named Keyhole Earth Viewer. It was created by Keyhole, Inc., which had been acquired by Google in 2004. The name "Keyhole" is homage to the $\mathrm{KH}$ reconnaissance satellites, the original eye-in-the-sky military reconnaissance system first launched in 1976. KML is an international standard of the Open Geospatial Consortium. Google Earth was the first program able to view and graphically edit KML files [15].

The KML file specifies a set of features (place marks, images, polygons, 3D models, textual descriptions, etc.) for displaying in Google Earth, Maps and Mobile, or any other 3D Earth browser (geobrowser) implementing the KML encoding. Each place always has a longitude and latitude. Other data can make the view more specific, such as tilt, heading, altitude, which together define a "camera view". A KMZ file consists of a main KML file and zero or more supporting files that are packaged using a Zip utility into one unit, called an archive. The KMZ file can then be stored and emailed as a single entity. When the KMZ file is unzipped, the main .kml file and its supporting files are separated into their original formats and directory structure, with their original filenames and extensions. In addition to being an archive format, the Zip format is also compressed, so an archive can include only a single large KML file. Depending on the content of the KML file, this process typically results in $10: 1$ compression (10 Kbyte KML file can be served with a 1 Kbyte KMZ file) [15].

All described above concerns that the use of KML can successfully solve problem of integrating animation of traffic flows into the Google Earth for demonstration purposes with high level or realism of environment in the study area. Also it is very important that almost everybody well knows GIS and is able to work with KML. 


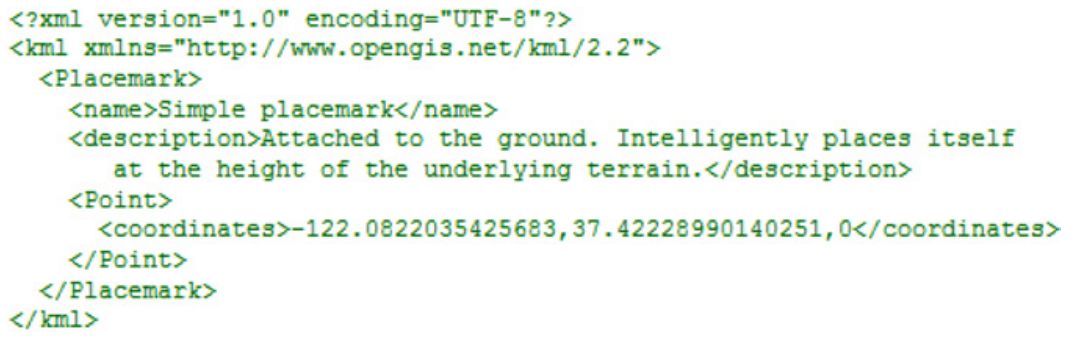

Figure 4. The example of simple KML file.

1. Configuration of the traffic simulation software add-in/external application

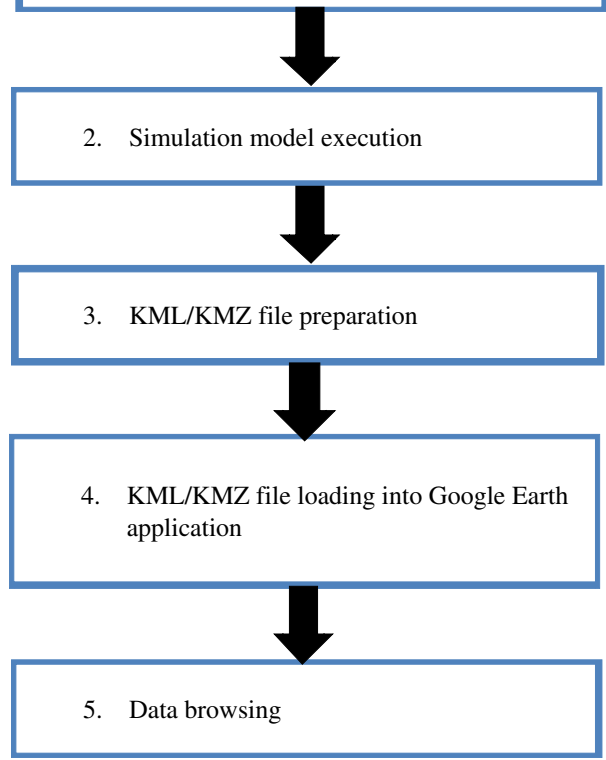

Figure 5. Workflow of visualisation.

\section{MICROSCOPIC TRANSPORT MODEL ANIMATION VISUALISATION}

The process of microscopic transport model animation visualisation and presentation could be described by 4 steps and the general workflows are presented on Figure 5.

The following comments could be given for each step of the workflow.

1. The first step of the workflow is a configuration of visualisation. The first step in the mentioned above workflow is very important for the next steps as it defines the way how KML/KMZ file will be created. First of all it is necessary to make a georeferencing. Georeferencing in this case means definition of longitude and latitude of simulation object. This information further will be used to map internal coordinates, used in simulation software to the Google Earth coordinates. In this stage it is necessary to bear in mind that for recalculation of internal coordinates of simulation software to the longitude and latitude should be used projections like Mercator or LKS-92. Further it is necessary to map vehicles classes used in simulation software to the vehicles 3D representation in format supported by Google Earth (at example COLLADA). 


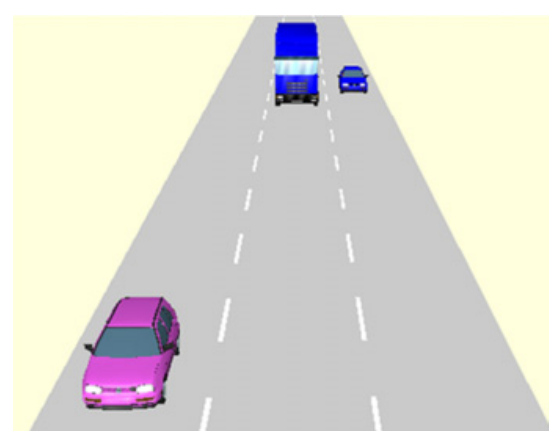

Figure 6. Visualisation in VISSIM.

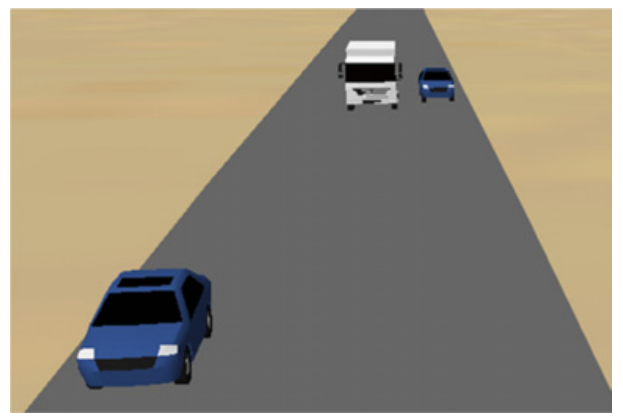

Figure 7. Visualisation in Google Earth.

2. Simulation model execution. This step includes execution of simulation model and tracing each vehicle position during executing. The position of vehicle will be presented by 3 coordinates. Using this data and georeferencing information completed in previous step, the longitude and latitude of the vehicle could be calculated in each simulation time step.

3. KML/ KMZ file should be constructed. Using collected information during simulation it is possible to generate KML/ KMZ. It must be noted that in this case it is better to use KMZ file as at the end we will get only one file, which will include one KML file and number 3D object files used for vehicles visualisation.

4. KML or KMZ file loading into Google Earth application or execution in browsers.

5. Data browsing. Data browsing can be done using Google Earth application or Google Earth plug-in for browsers. Of course, the third party software, which can read KMZ or KML files, could be used.

Also it must be noted that there are two ways how technically visualisation software could be organised.

1. Using external application written in any programming or scripting languages. This possibility could be used, because VISSIM have a COM interface. The advantage of this decision is that you can use any programming language, which you know. The disadvantage is slow communication via COM interface with VISSIM.

2. Using VISSIM add-ins written on Python scripting language. The advantages - module integration into VISSIM, so you can call it in any time, without launching any external application; the communication speed with VISSIM is higher than in the first case. The disadvantage - limitation on using only Python as scripting language. 


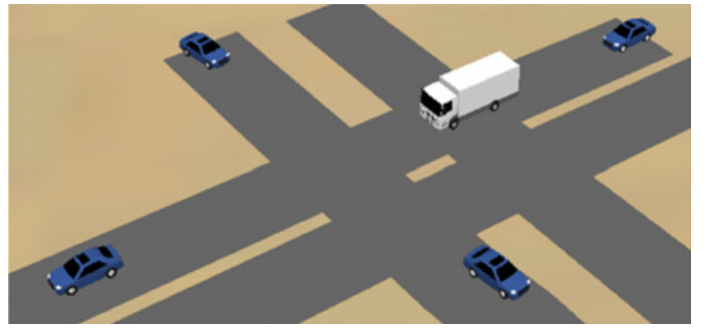

Figure 8. Visualisation of crossroad in VISSIM.

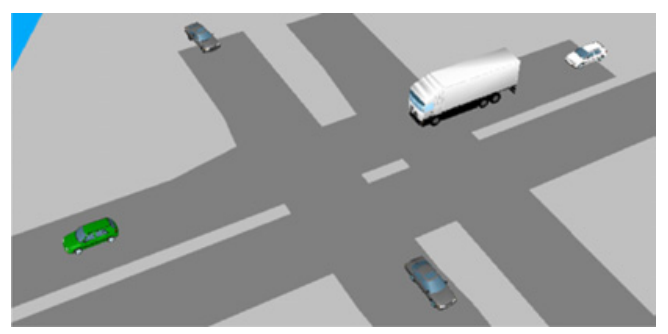

Figure 9. Visualisation of crossroad in Google Earth.

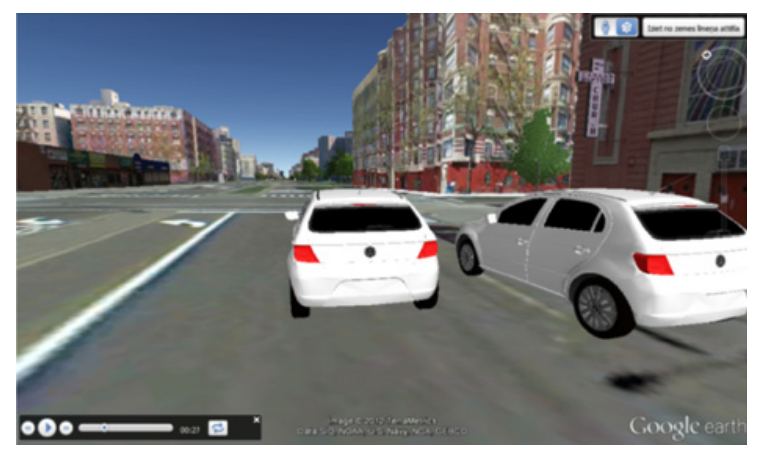

Figure 10. Visualisation results.

\section{PROTOTYPING AND CASE-STUDIES}

According to the described above concepts of traffic simulation software for visualisation by application of KML a prototype of VISSIM external application has been developed and tested to the ability fit necessary functional requirement to the visualisation. The prototype of software is developed using Visual Basic, VISSIM 5.2, Google Earth and KML. Using developed prototype the following case studies are prepared and further will be demonstrated and commented.

Case-study 1. The case-study presents the results on visualisation of straight vehicles' movements in VISSIM and in Google Earth application (see Figures 6 and 7).

The developed software launches VISSIM and does simulation step by step. On each step the coordinates of vehicles in VISSIM are obtained and converted to the longitude and latitude. As time 
between steps is a very small value, equal to $1 \mathrm{~s}$, the movement of vehicles in Google Earth is very smooth. This fact is particularly important for visualisation.

Case-study 2. The case-study demonstrates more complex object for visualisation. The complexity of this example connected with turning movement of vehicles. The 3D object, which represents vehicles during visualisation, must be rotated according to the turning movement. It is of great importance for quality of visualisation.

Case-study 3. Final example shows the visualisation of VISSIM animation in 3D environment. From Fig. 10 could be seen a view of vehicles in 3D environment, which exists in Google Earth application.

\section{CONCLUSIONS}

One of the advantages of the simulation is a possibility to present process and data in visual form via animation and graphical representation in 2D and 3D format. For transport modelling the main goal of visualization is the presentation of results to non-professional auditorium in credible manner.

In general modern professional simulation tools for transport modelling support 2D and 3D representation construction of the models. But usually the development of realistic 3D environment requires a lot of time.

The possibility of using KML (Keyhole Markup Language) for 3D visualisation of the animation data from traffic simulation software is demonstrated in the given article. The examples of using KML for visualisation are presented using PTV VISION VISSIM software, Visual Basic and Google Earth application. The selected tools are subject of availability and experience of use, because in general proposed approach could be realised using any GIS, which supports KML and any traffic simulation software, which has integrated programming features. Such approach of animation visualisation can help to decrease development time of realistic 3D environment. Also, it gives a possibility easy to share visualisation among users via Internet.

The development of the examples revealed some problems, which could be mentioned here:

- For the object positioning longitude and latitude coordinates must be used. Despite the fact that most of simulation software has a possibility to use different types of coordinates, many transport models are constructed in the scale of software coordinates. This problem was has been solved by development of small module, which maps software coordinates with longitude and latitude. Of course, this operation requires manual data entering. This point is of great importance because the incorrect georeferencing can produce not correct visualisation view (see examples, vehicles will be outside of road, etc.).

- Analysis of 3D objects (buildings) in Google Earth shows that 3D representation of buildings has only big cities located in the USA. In other cases the 3D environment is fragmented. Of course, this point put in trouble all concept of visualisation, but still we are very optimistic as number of 3D models in Google Earth is growing extremely.

\section{References}

[1] A. van den Brink, R. van Lammeren, R. van der Velde, S. Däne, "Imaging the future: geovisualisation for participatory spatial planning in Europe". Wageningen. Academic publishers, Mansholt publication series 3. 169-174 (2007).

[2] J. Banks, J.S. Carson II, B.L. Nelson, D.M. Nicol. Discrete-Event System Simulation. 3th Edition. - Prentice Hall 594 (2001).

[3] Prepared by the AASHTO Task Force on Environmental Design "Visualization in Transportation". American Association of State Highway and Transportations Officials (2003).

[4] R. Chapleu "Visualizations of the urban transportation reality: some key views". 10th International Conference on Travel Behaviour Research, Lucerne, Suisse, 30 (2003) 
[5] R. Chapleau, C. Morency. "Dynamic Spatial Analysis of Urban Travel Survey Data using GIS”. A paper presented at the ERSI User Conference in 2005. http://www10.giscafe. com/link/display_detail.php?link_id=13155 [Last accessed: 10 May 2012].

[6] S. Schönfelder, K.W. Axhausen. Structure and innovation of human activity spaces, Arbeitsberichte Verkehrs- und Raumplanung, 258, IVT, ETH Zürich, Zürich (2004).

[7] Y.B. Shrinivasan and M.J. Kraak. "Visualization of spatio - temporal patterns of public transport data”. In: Proceedings of the 22nd international cartographic conference: mapping approaches into a changing world, A Coruña, Spain. International Cartographic Association (ICA), (2005).

[8] C. Hixon, "Visualization Issues for Transportation Agencies: Approaches and Challenges". TRNews, September-October, 11-14 (2007).

[9] T.J. Kimpel. Data Visualization as a Tool for Improved Decision Making within Transit Agencies. Final Technical Report TNW2006-14. A report prepared for Transportation Northwest (TransNow), University of Washington, Seattle, WA., 112 (2007).

[10] D. Walker, "Visualization as Common Language for Planning". TRNews, September- October, 7-10 (2007).

[11] J. Ortuzar, L. Willumsen, Transport Modelling. 3-rd edition. Wiley: NY, 499 (2005).

[12] M. Savrasovs, A. Rudzitis, "Macroscopic transport model data 3D visualization using KML". In Proceedings of $10^{\text {th }}$ International Conference, Reliability and Statistics in Transportation and Communication, 440-446 (2010).

[13] I. Yatskiv, E. Yurshevich, M. Savrasov, "Practical aspects of modelling in the transport node reconstruction in Riga", In Proceedings of 23rd European Conference on Modelling and Simulation, Madrid, Spain, 295 - 300 (2009).

[14] M. Kutz, "Handbook of transportation engineering”, McGraw-Hill Professional, 1000 (2004).

[15] T. Wilson, “OGC KML 2.2.0”, Document \#07-147r2, Open Geospatial Consortium. 2008. Available online: http://www.opengeospatial.org/standards/kml/ [Last accessed: 10 May 2012]. 